\title{
=ALTERIDAD Investigación y aprendizaje: Retos en Latinoamérica hacia el 2030
}

\section{Research and learning: Challenges in Latin \\ America towards 2030}

D Dr. Julio Juvenal Aldana-Zavala es docente e investigador de la Universidad Nacional Experimental Francisco de Miranda y Red de Investigación Koinonia (Venezuela) (julioaldanazavala@gmail.com) (https://orcid.org/0000-0002-7934-9103).

(D) Dr. Patricio Alfredo Vallejo-Valdivieso es docente e investigador de la Universidad Técnica de Manabí (Ecuador) (patricio_2871@yahoo.es) (https://orcid.org/0000-0003-3248-7864).

(D) Dr. Josía Isea-Argüelles es docente e investigador de la Universidad Nacional Experimental Francisco de Miranda y Red de Investigación Koinonia (Venezuela) (josiaisea@gmail.com) (https://orcid.org/0000-0001-8921-6446)

Recibido: 2020-01-31 / Revisado: 2020-10-21 / Aceptado: 2020-11-26 / Publicado: 2021-01-01

\section{Resumen}

La educación ha tenido un giro epistémico en su accionar pedagógico, transcendiendo del modelo tradicional enfocado en el docente, hacia uno focalizado en el estudiante. En paralelo, la investigación se ha fomentado hacia nuevas vertientes metódicas, siendo un factor clave en el aprendizaje. Hoy día es esencial la fusión del aprendizaje e investigación con la función de generar conocimientos desde nuevos ángulos educativos. Además, la UNESCO y otros entes enfocados a la educación, han propuesto la conformación de un modelo educativo para aprender a lo largo de la vida. Es así que la presente investigación presenta los alcances en investigación y aprendizaje en Latinoamérica, para tal fin se planteó como objetivo central: Analizar la investigación y aprendizaje como retos en Latinoamérica hacia el 2030, en este sentido se empleó una metodología descriptiva documental con un diseño bibliográfico, teniéndose como muestra poblacional, la revisión de 52 artículos de revistas indizadas en Scopus, Web of Science, Scielo, Redalyc, Latindex Catálogo 2.0. Esto con la finalidad de sistematizar propuestas de investigadores, identificándose factores pertinentes hacia la consecución de una educación transversal como medio para el logro de los ODS. Entre las conclusiones se tiene la necesidad de formar en una epistemología posibilitadora de una ecología de saberes en procura de contar con instituciones educativas, sostenibles y productivas como eje medular de la sociedad global.

Descriptores: Investigación pedagógica, organización, aprendizaje activo, educación alternativa, política educacional, estrategias educativas.

\section{Abstract}

Education has had an epistemic turn in its pedagogical action, going from the traditional model focused on the teacher, to one focused on the student; at the same time, research has been encouraged towards new methodical aspects, being a key factor in learning. Today, it is essential to merge learning and research with the function of generating knowledge from new educational angles. UNESCO and other entities focused on education have proposed the creation of an educational model for lifelong learning. Thus, this research presents the scope of research and learning in Latin America, for this purpose it was proposed as a central objective:To analyze research and learning as challenges in Latin America towards 2030, in this sense a descriptive methodology was used with a bibliographic design, taking as a population sample, the review of 52 articles from journals indexed in Scopus, Web of Science, Scielo, Redalyc, Latindex Catalog 2.0. This with the purpose of systematizing researchers' proposals, identifying pertinent factors towards the achievement of a transversal education as a means for the achievement of the SDG. Among the conclusions is the need to form an epistemology that makes possible an ecology of knowledge in order to have educational, sustainable and productive institutions as the core of the global society.

Keywords: Educational research, organization, activity learning, alternative education, educational policy, educational strategies.

Forma sugerida de citar: Aldana-Zavala, J., Vallejo-Valdivieso P., \& Isea-Argüelles, J. (2021). Investigación y aprendizaje: Retos en Latinoamérica hacia el 2030. Alteridad, 16(1), 78-91. https://doi.org/10.17163/alt.v16n1.2021.06 


\section{Introducción}

La educación global transita entre la sociedad de la información y transcender a la del conocimiento; ambos involucran lo tecnológico e informático, varía en cuanto se plantea que el estudiante debe transformar la información recibida (sociedad del conocimiento), tal como lo propone la UNESCO (2005). El aprendizaje desde lo abordado, involucra fomentar en el estudiante un proceso integral de interconexión con las múltiples realidades en donde se desenvuelve, siendo necesario un reconocerse a sí mismo y al otro como sujetos cognoscentes e inteligibles emocionales para construir desde la intersubjetividad un ambiente de respeto mutuo, valoración de las capacidades y trabajo cooperativo para la construcción del conocimiento en razón de conformar escenarios educativos donde la ética sea transversal en el razonamiento de comprender a la persona como un ente transcendental para la construcción de una mejor sociedad. A partir de lo planteado, la investigación tiene por objetivo central, analizar la investigación y aprendizaje como retos en Latinoamérica hacia el 2030.

\section{Método}

La metodología empleada es de tipo descriptivadocumental con un diseño bibliográfico, lo que permitió escrutar 52 artículos de revistas indizadas en Scopus, Web of Science, Scielo, Redalyc y Latindex Catálogo 2.0 con el fin de conocer la tendencia, retos, debilidades y fortalezas de la educación latinoamericana en función de la investigación y aprendizaje hacia el 2030; aplicándose la técnica de análisis de contenido para extraer las ideas más relevantes planteadas por los investigadores y así, construir un cuerpo teórico como aporte a la generación de conocimientos en actualización del estado de la cuestión, contribuyendo a generar investigaciones posteriores desde una óptica experimental o cualitativa.

\section{Resultados y discusión}

Se presentan cuatro áreas de análisis en conformidad de brindar respuesta al objetivo central, entre las cuales se encuentran: Educación y objetivos de desarrollo sostenible; La planificación centrada en la investigación; Docente investigador; Hacia un abordaje epistemológico educativo 2030.

\subsection{Educación y objetivos de desa- rrollo sostenible}

La educación al ser catalogada en factor transversal juega un papel primordial en la efectividad de lograr los objetivos de desarrollo sostenible, no puede percibirse como un ente aislado o dicotómico. Es necesario transcender hacia un modelo integrador donde los actores educativos tengan la oportunidad de construir conocimientos holísticos e interrelacionados con el mundo global y las múltiples necesidades a superar para lograr una sociedad de paz con base en el progreso productivo. Al respecto Murillo y Duk (2017), destacan el apremio del tiempo para cumplir con los ODS, requiriéndose estructurar urgentemente planes estratégicos en los entes educativos y de investigación, concatenados para optimizar recursos en la consecución de una educación centrada en la investigación para aprender a lo largo de la vida. Mientras que Cosme-Casulo (2018), complementa al señalar a la universidad como institución vital para tal fin, debido a su rol de formador de formadores e investigadores, profundizando la vinculación con las comunidades en razón de intercambiar saberes para la transformación mutua.

Ambos autores concuerdan en la necesidad de una educación basada en cambios de paradigmas desde la formación universitaria y las modalidades de investigar, viéndose involucrados los centros de investigación como factor gerencial para la proyección de investigaciones pertinentes al devenir 2030, incluyendo a las organizaciones públicas y privadas, en un trabajo mancomunado para el aporte de resultados sig- 
nificativos al logro de la supresión de la pobreza, concordando los autores en una correlación directa entre los objetivos de desarrollo sostenible 4 y 16.

La educación al no contar con el debido financiamiento entra en conflicto sobre el rol social que juega, es imprescindible preguntar si: ¿Es un bien común? o ¿un factor para el desarrollo económico? ¿indicador para el crecimiento social? y, ¿qué es realmente la educación y qué papel persigue de cara al 2030? Locatelli (2018), colabora en descubrir las respuestas al plantear la necesidad de "desarrollar sistemas más sostenibles” (p. 194). En una sociedad donde la deserción escolar es una amenaza para las clases sociales de mayor vulnerabilidad, se hace necesario generar una estructura escolar sostenible, lo cual implica flexibilizar los currículos para la integración e inserción laboral de los estudiantes, posibilitando complementarse entre lo económico y académico. En este sentido, Agosto et al. (2018), indican la necesidad de políticas de atención de los jóvenes, cooperando en su crecimiento integral, mediante la generación de una visión de futuro basada en proyectos de vida, para lo cual la educación no debe basarse en el mero modelo de inclusión, sino, complementarse con una epistemología compleja de abordaje de la realidad social como eje medular para configurar un racionamiento reflexivo del actuar para el bien de la sociedad.

El sistema educativo debe transitar al ritmo que lo hacen las sociedades, la dinámica social es cambiante y cada día surgen expectativas sobre todo en la población joven sobre lo que será su futuro socioeconómico, en un mundo donde lo tecnológico abre brechas entre quienes tienen o no acceso a la misma; es impensable que la escuela y la universidad del futuro próximo debe ser igual hasta lo que ahora conocemos y manejamos. Entonces, ¿cómo debe ser la educación de cara al 2030?; necesariamente integrativa de todos los factores que conforman la sociedad, conformando planes estratégicos de crecimiento integral en el cual se plantee el hacia dónde se pretende ir y cómo se va a lograr, al ser la educación el eje medular. Vera-del Carpio (2015), amplía diciendo que es necesario la unión de lo público con lo privado para ejecutar acciones concretas desde la educación al logro de una sociedad sostenible, con base en la formación preventiva de la salud, ecología y ambiente, aristas necesarias para contar con una sociedad saludable en todos sus ámbitos, optimizándose recursos para ser invertidos en educacióninvestigación, además de propiciar una cultura ciudadana de respeto para la convivencia entre el ser humano y factores bióticos-abióticos.

Azorín-Abellán (2017), indica el reto global de propiciar una educación para todos, eficaces e inclusivas, donde se exige desde los organismos multilaterales articular la inclusión, calidad, eficacia y equidad, como indicadores que permitan transversalizar la educación en pro de contribuir al logro de los objetivos de desarrollo sostenible, lo cual implica redescubrir el rol social de la educación como factor neurálgico en consideración de generar la sinergia de los diversos actores sociales con la finalidad de consolidar una actuación que permita el logro efectivo de los cuatros indicadores mencionados como elemento central para contar con una sociedad basada en la alteridad educativa.

\subsection{La planificación centrada en la investigación}

Una educación transversal e integrativa de procesos que permita concatenar acciones en pro de trabajar en la consecución de los 17 objetivos de desarrollo sostenible como acción concreta para la transformación social de los pueblos en favor de erradicar la pobreza, debe estar apuntalada en la mejora permanente de los estilos de cómo se promueve el aprendizaje en los ambientes educativos y contar con la investigación como eje fundamental para tal fin. De ese modo, el currículo debe estar concebido desde una intencionalidad investigativa donde los actores educativos trabajen cooperativamente para el logro 
de metas propuestas, es así que los docentes deben planificar desde una visión centrada en la investigación-acción como eje alternativo para superar el modelo dicotómico de educar e investigar, propio del enfoque disciplinar (Pérez-Van Leenden, 2019).

La planificación centrada en la investigación como modelo pedagógico invita a considerar el aporte y potencialidades de todos los actores involucrados en el proceso de aprendizaje, esto implica involucrar a la comunidad educativa en su contexto social, así cada uno puede brindar aportes significativos en la construcción de currículos donde se aprenda a pensar, construir, resolver problemas de interés social e innovar; trabajando en el enfoque por proyectos, así como otros donde el estudiante tome un rol protagónico en aras de articular acciones que favorezcan el conocimiento para la vida, considerándose esto como un criterio de calidad educativa, es necesario contar con programas estratégicos enfocados a guiar la educación de cara al 2030, esto un obstáculo para el logro de una educación inclusiva tal como lo señala (Meléndez-Rojas, 2017), presupuestando una educación creativa e innovadora a largo plazo, formando para ser eficientes en tecnología en armonía con el medio ambiente.

Uno de los aportes de la planificación centrada en la investigación es que permite concebir la educación desde la centralidad del estudiante y no desde el docente, con lo cual se pueden incluir diversos enfoques pedagógicos basados en la cosmovisión del aprendizaje dinámico, flexible y reflexivo, apoyado en las tecnologías de información y comunicación, contextualizado a las múltiples necesidades sociales (Peche-Cruz \& Giraldo-Supo, 2019). Ante esta postura pedagógica, Molina-Naranjo et al. (2018), destacan la necesidad de formar a los futuros docentes desde un currículo transversal en lo "epistemológico, axiológico, investigación, formación, competencias para la integración de las TIC y competencias profesionales generales" (p. 162); lo cual debe converger en el enfoque centrado en el estudiante, propiciándose progresivamente un cambio en la praxis del profesional educativo, posibilitándose la comprensión, para asumir nuevas posturas pedagógicas donde se transcienda el statu quo, favoreciéndose esta acción, desde la formación-evaluación continua como parte del proceso educativo (Huapaya-Capcha, 2019).

En virtud de lo abordado, es ingenuo pensar que, con el solo cambio de planes en lo documental sin contar con la debida sensibilización y formación a los actores educativos, estos optarán por asumir asertivamente los cambios paradigmáticos propuestos. De ahí un reto intrínseco en el logro de construir una planificación centrada en la investigación como estrategia transversal para el logro de una educación hacia el 2030. Otro reto es fundamentar la investigación como un proceso atractivo para los estudiantes, sin que esto implique la pérdida del carácter científico de la misma, ante lo cual, Loli-Ponce (2015), indica que los estudiantes manifiestan no tener el tiempo necesario para hacer investigaciones, aunque están conscientes de la importancia que reviste la misma para el aprendizaje y para el conocimiento, por cuanto el currículo disciplinar fragmentado, al percibir la investigación como un producto final del estudiante, no se contextualiza a lo largo del entramado curricular como un ente integrado a la formación.

Esto indica que el enfoque de planificación centrado en la investigación debe ser asumido desde una reconstrucción del currículo, por cuanto no podría trabajarse con una visión tradicional y emergente a la vez en la educación, se necesita de una transposición educativa para conformar una educación de calidad en la praxis y no en una mera retórica de los actores sociales. En este sentido, Calvo (2013), comenta que la formación docente debe estar planificada en función de la participación de los diversos factores sociales, lo cual implica una obligatoria concatenación entre la educación y las diversas instituciones públicas y privadas, planteándose un programa para todos en favor de una educación inclusiva. 
El involucramiento de los actores sociales para que sea efectivo, a juicio de Carrillo-Flores (2016), pasa por develar lo oculto de la educación, es decir, las ideologías que han estado implícitas en la misma como un modo de sincerar el trabajo hacia la transcendencia de la inclusión como un ente utópico, dado que la autora destaca que los principios y las intenciones educativas no son neutrales desde una perspectiva política. Es necesario realizar un paréntesis para señalar que cuando se plantea la educación inclusiva en el actual documento, se hace pensando en la posibilidad que todos los actores sociales tengan acceso a la educación distanciándose del término inclusiva como algo exclusivo de las personas con discapacidad- por cuanto existen discapacidades sociales que excluyen muchas veces, tal como promueve CornejoEspejo (2019), al concebir la inclusión como un reconocimiento ético de la realización humana, subrayando la importancia de la deconstrucción de los paradigmas que promueven la exclusión, es necesario retomar la esencia fundamental de la educación como un derecho al cual deben tener acceso todas las personas.

La planificación centrada en la investigación cuando se asume como un hecho complejo e inclusivo donde los actores educativos tienen la posibilidad de ser protagonistas y no espectadores; pronostica un cambio paradigmático donde la ciencia sin perder su rigurosidad se hace accesible. El aprendizaje contribuye a formar ciudadanos críticos-reflexivos, teniendo en consideración el término "bioresistencia" de Aya-Velandia (2018), quien explica que "es una manera de asumir los conocimientos científicos y aplicarlos en la cotidianidad" (p. 205). Se redescubre el rol de la persona como un ser integral donde los diversos factores exógenos influyen para la conformidad de un sujeto cognoscente al servicio de la sociedad.

Un sujeto cognoscente implica una relación con otros sujetos cognoscentes, siendo necesario transcender el paradigma basado en el sujeto cognoscente y un objeto cognoscible que ha estado permeando la investigación educativa tradicionalmente, posición apoyada por Cúnico et al. (2018). Estos autores cuestionan "el paradigma epistemológico dominante, alentando y fomentando nuevas formas de investigación en psicología" (p. 213); plantean desde la piscología, ciencia coadyuvante de los procesos pedagógicos, pero que además en una visión multi e interdisciplinar hacia donde se orienta la educación 2030, debe estar presente la investigación desde lo complejo.

No debe percibirse la investigación como un mero acto procedimental, debe existir una conciencia epistemológica por parte del investigador o investigadores en la que sea imprescindible abordar el conocimiento desde la filosofía como eje transversal en la educación. Esto contribuye a comprender los diversos enfoques investigativos y curriculares, generándose mejor comprensión del fenómeno educativo, ante lo cual es necesario fomentar la competencia epistemológica como arista transversal para imaginar la realidad educativa-investigativa desde una óptica compleja de la sociedad, involucrando la inclusión metodológica de nuevas posturas para la realización de la investigación (Aldana-Zavala, 2019).

Esta competencia se extrapola al investigador educativo desde el contexto inicial al universitario, esto contribuye a fomentar un abordaje epistémico-metodológico coherente y lógico del accionar investigativo en ambientes de aprendizaje. Así mismo, Borrero (2019) contribuye en complementar lo expuesto al indicar que los docentes tienen la disponibilidad de conocer nuevos modos de hacer investigación, pero presentar debilidades para la realización de investigaciones desde los diversos métodos, para lo cual requieren formación permanente, así como el apoyo de la gerencia educativa; además, es necesario que las instituciones educativas tengan planes de operatividad educativa concatenados a los de desarrollo socio político local y nacional.

Tal formación debe permear los estudios de posgrado donde los docentes e investigadores proyectan mejorar su profesionalización, terreno fértil para el abordaje de nuevos modos de for- 
mar e investigar como referencia en este ámbito se tiene en consideración a Proestakis-Maturana y Terrazas-Núñez (2017). Estos autores destacan el compromiso de abrir espacios de reflexión y discusión entre investigadores, estudiantes y docentes, para romper la estructura disciplinar; se destaca la necesidad desde los estudios doctorales, transcender en la construcción de investigaciones con perspectivas emergentes, involucra la visión de quién dirige el programa, así como de los asesores, integrantes de una reconstrucción epistémica del currículo. En complemento, DíazBazo (2017), comenta la importancia que tiene la investigación-acción en la implementación de algunas áreas temáticas en comparación con otras, lo que deja claro que estamos transitando en una sociedad pluripolar epistemológica y metódica, en donde es necesaria la integración y complementación, para lograr acuerdos en mejora de la educación, quizás lo transdisciplinario o complejo sea una opción; lo importante de destacar es el rol del docente como investigador.

\subsection{Docente investigador}

El docente investigador deja de lado la formación continua con la finalidad de acumular conocimientos y transmitirlos, para transcender a la construcción de los mismos. Esto implica un giro de $180^{\circ}$, por cuanto la constante indagación investigativa lo erige como una persona autodidacta en capacidad de aprender haciendo y posibilitando reflexionar en el día a día para motivarse a superarse a sí mismo en procura de ser mejor persona y, por ende, investigador. Muñoz-Martínez y Garay-Garay (2015), advierten que "la investigación educativa es una formación continua” ( $\mathrm{p}$. 398), por lo tanto, en un mundo dinámico se hace necesario un docente investigador con la finalidad de adoptar soluciones a los múltiples retos sociales a los cuales se enfrentan, esto implica modelar en los estudiantes el rol de investigador como elemento de aprendizaje para la vida.

Lo expuesto invita a reflexionar de modo constante en que es importante aprender y cómo aprenderlo, por cuanto existe una tendencia de eliminar de los pensum de estudios, todo aquello que no se perciba como utilitario para el crecimiento económico-tecnológico de la sociedad, ante lo cual surge una corriente discriminatoria del pensamiento humano, contribuyendo indirectamente en la aparición de sociedades de masas. Escámez-Sánchez et al. (2017) complementan reflexionando sobre la urgencia de educar para la conformación de una sociedad sostenible - en ese sentido- lo económico es vital para el crecimiento humano y educativo, pero no debe verse como el fin, sino, como un medio.

La importancia de compaginar en la formación de valores humanos, sociales y profesionales para formar para la vida con conciencia de la coexistencia humana con otras especies en el planeta tierra; así Rodríguez-Fiallos et al. (2019), proponen la investigación como una vía para la autorrealización y autonomía de la persona, necesidad que el docente investigador tenga una formación que le permita reconocer el valor de la persona en consonancia de aplicar estrategias pedagógicas flexibles y dinámicas, de acuerdo con el contexto social. Rivas-Tovar (2011) propone nueve competencias para la producción científica investigativa como eje transversal de la educación.

Transitar hacia una visión compleja del accionar investigativo es una tarea del día a día al cual se enfrentan los investigadores educativos, esto involucrar y deconstruir para construir nuevos estadios de hacer investigación. FernándezHernández y Cárdenas-Berrio (2015), amplían al señalar que la "poca visión dialéctica de algunos estudiantes ante el proceso de investigación, la carencia de métodos y herramientas más allá de los asociados a la investigación cuantitativa" ( $p$. 45); constituyéndose lo referido en un indicador de la necesidad de contar con docentes investigadores que cuestionen su realidad investigativa, atrofien lo cotidiano y en una emergente dialéctica redescubran el accionar investigativo como un medio para el crecimiento integral, fomentando esta aptitud en sus estudiantes para constituir equipos de investigación para la construcción 
permanente del conocimiento. Buendía-Arias et al. (2018) consideran pertinente superar la dicotomía entre investigación y práctica pedagógica, lo cual contribuirá en una educación para la reflexión, indagación y crecimiento autónomo de las personas.

El docente investigador se encuentra en la era educativa de enseñar a investigar, proceso que permite incorporar subprocesos como la lectura, indagación, análisis, reflexión, discriminación de información y aplicación de técnicas para el procesamiento de información, argumentación y uso de tecnología, entre otros, que contribuyen en formar significativamente para el aprendizaje a lo largo de la vida. Por otro lado, García et al. (2018), plantea que, con un buen docente investigador, tiende a mejorar las competencias investigativas en los estudiantes, mejorándose además su capacidad intelectual, siendo fundamental para tal fin, hablar y escribir bien la producción científica, por cuanto la sociedad global, confronta problemas para la lectura y apreciación crítica de la realidad social, el currículo educativo debe asumir este reto como parte de su cotidianidad pedagógica.

Destacándose la importancia que deben brindar las instituciones educativas a la investigación, sobre todo al papel fundamental del docente investigador, por cuanto lo contrario implica una subestimación de la investigación que va en quebranto de la calidad educativa y del aprendizaje, tal como lo advierte Pérez (2017). Así mismo, García-Gutiérrez y AznarDíaz (2019), confirman la visión de profundizar en la lectura, escritura y competencias investigativas como esencia formativa del futuro docente, reflexionándose así, sobre el papel que juega la universidad en la formación de los futuros educadores, quienes deben estar en consonancia con ejercer profesionalmente para brindar respuestas a las múltiples realidades sociales, para lo cual deben formarse en competencias investigativas para accionar asertivamente en tal fin.

Esto implica que las universidades en Latinoamérica deben redescubrir la investiga- ción como un eslabón para elevar la calidad educativa; Murillo y Martínez-Garrido (2019) concuerdan que la investigación tiende a manejarse desde la polarización de lo cuantitativocualitativo. A partir de lo descrito se involucra que en Latinoamérica aún falta por profundizar en la generación de investigaciones desde nuevos enfoques metodológicos, de allí que invitan a sistematizar experiencias pedagógicas e investigativas con la finalidad de concebir nuevos estilos de investigación pertinentes en la conformación de una educación de calidad. La actividad investigativa puede tener en cuenta métodos clásicos de lo cualitativo, pero poco abordados como punto de partida, siempre y cuando estos resulten pertinentes al accionar pedagógico del docente investigador. A juicio de HuchimAguilar y Reyes-Chávez (2013), la investigación biográfica-narrativa puede coadyuvar con lo descrito, requiriéndose formar en competencias comunicativas para ser efectivos en la realización de este tipo de investigación.

Otro enfoque que debe ser revisado para conciliar un docente investigador pertinente a los retos 2030, es lo referido al liderazgo educativo. Villa-Sánchez (2019), explica la importancia que tiene el liderazgo educativo como promotor de cambios significativos en la institución educativa, así como en los modelos de cómo se trabaja pedagógicamente, por cuanto un líder transformador encausa a aprender nuevas tendencias metodológicas en favor de promover cambios pedagógicos. El compromiso de la gerencia educativa en el accionar de políticas institucionales, ejerce una motivación en el liderazgo docente para conformar un trabajo cooperativo, dinámico y flexible, en razón de construir un aprendizaje pertinente desde el rol del docente investigador como líder transformador, situación planteada por Morgado et al. (2019), al concebir el rol del directivo desde una visión transformacional como necesaria para lograr cambios en la institución.

Trabajar desde la concepción de un liderazgo con base en la inteligencia emocional contribuye a permear al logro de metas en la 
institución educativa. Maya et al. (2019), sostienen que una praxis profesional centrada en lo humano, aporta sinergia al trabajo cooperativo. Izquierdo-Rus et al. (2019), destacan que, en el trabajo cooperativo, los estudiantes tienden a tener dos problemáticas: la primera que no les agrade el trabajo a realizar, y la segunda con quién lo van a realizar. La investigación educativa es un trabajo cooperativo desde la perspectiva epistemológica sujeto-sujeto, invita al docente a reflexionar sobre su liderazgo en el accionar pedagógico, el cual debe ser motivador y conciliador, en procura de equilibrar las emocionalidades de los estudiantes en favor de investigar para aprender a lo largo de la vida. García-Garnica y Martínez-Garrido (2019), apoyan la necesidad de contar con liderazgo escolar asertivo para la consecución de objetivos institucionales, como factor previo para establecer un efectivo aprendizaje cooperativo.

La investigación vista desde lo planteado, debe apoyarse en nuevas formas de experimentar la clase por parte de los estudiantes, donde estos tengan la posibilidad de tener mayor protagonismo y en donde se vean involucrados procesos investigativos. Esta visión no escapa al docente quien debe manejar nuevos estilos didácticos para contribuir en la transcendencia del modelo tradicional a enfoques que se encuentran en concordancia con una educación al 2030, insistiéndose en la formación permanente e integral del docente para asumir asertivamente tales retos. Del Arco-Bravo et al. (2019), consideran para tal fin, la intensificación del enfoque centrado en el estudiante, por cuanto este permite incentivar la motivación, especialmente entre los de mayor edad.

\subsection{Hacia un abordaje epistemoló- gico educativo 2030}

Como se ha abordado en líneas anteriores, es necesario el fomento de una competencia epistemológica en los investigadores docentes con la finalidad de cuestionar la realidad desde una perspectiva filosófica de la realidad, de cara al 2030. La educación latinoamericana se encuentra frente al reto de transcender la racionalidad cartesiana en asunción de asumir una postura epistémica que le permita identificarse para el trabajo pertinente y contextualizado a su realidad social. Pero, ¿cuál es esa epistemología? Podría indicarse que se mueve en dos mares: uno desde la visión compleja eurocéntrica emergida del conocimiento promovido desde la física cuántica versus al de la física clásica; un segundo, relacionado con una mirada desde lo decolonial, pero es acaso posible seguir en una lucha dicotómica o es viable la fusión para construir una visión inclusiva desde el saber científico.

Collado-Ruano (2017a), destaca la simbología de una sociedad en construcción, adherida desde diversos ángulos epistémicos, siendo Latinoamérica una fusión intercultural y, por ende, mestiza en lo epistemológico — es posible experimentar metódicas pedagógicas para afianzar una identidad científica en lo diverso y complejo- favoreciendo un ecosistema de saberes entre las culturas que habitan un territorio.

Es una eterna construcción hacia lo intercultural lo que permitirá compaginar un accionar epistemológico donde se valore la investigación educativa como centro para conformar conocimientos conducentes a una educación inclusiva 2030. La lucha de poderes entre las personas y colectivos, institucionalizados en las instituciones educativas que se aferran a un saber-poder como primogénito y superior en detrimento de otras opciones epistémicas y metódicas es un reto cultural a transcender para lograr el equilibrio de la sostenibilidad de los saberes. En complemento, Collado-Ruano (2017b), propone que "hacer frente a los desafíos de los ODS requiere crear una ecología de saberes que reintegre los diferentes conocimientos y dimensiones humanas" (p. 246).

La ecología de saberes permite integrar conocimientos ancestrales, científicos, cotidianos o de la sabiduría popular, para confluir en un equilibrio del conocimiento pertinente a 
proporcionar la ruptura de las perspectivas polarizadas de la educación planificada en función de las ideologías dominantes del poder político. Es necesario confrontar la realidad desde el aporte de los diversos actores sociales en la construcción de políticas educativas en razón de convivir desde la educación como un fenómeno de convivencia colectiva (Collado-Ruano et al., 2018), constituyéndose competencias pedagógicas-investigativas en coalición de las ciencias ancestrales y ciencias científicas.

El buen vivir puede ser una opción sostenible del equilibrio humano por cuanto procura convivir en una postura media entre la riqueza y la pobreza, donde se puedan cultivar valores éticos como expresión de interrelación entre los seres vivos y no vivos del planeta (ColladoRuano, 2016), siendo necesaria la revisión del modelo económico y su relación con las leyes biofísicas de la naturaleza.

Fernández-Galindez (2019) y AlfaroMardones et al. (2015), cooperan con lo planteado al advertir sobre la importancia de velar por la transformación y la autorreflexión como medio de sanación emocional-espiritual, como punto de referencia para lograr cambios en la educación y en lo social, lo cual implica que si no se forma para asumir la integralidad como expresión pedagógica, las concepciones epistemológicas de diferente planteamiento a la educación tradicional, pueden verse truncadas en su efectividad, colocando en peligro la opción de lograr la sostenibilidad como ejercicio para el buen vivir de la sociedad global. Acosta (2016) destaca la construcción transdisciplinar de la universidad para formar desde la coexistencia entre lo científico y las dimensiones que engloban al ser humano. Es una visión para confluir en una ecología de saberes y convivencia en la construcción de una sostenibilidad en el quehacer diario de los actores educativos, donde la formación sea concebida en edificar ciudadanía planetaria, como espacio de convivencia basada en el auto-respeto, entre lo sociológico y natura- leza, tal como destacan Aldana-Zavala y ColinaYsea (2019).

Desde la integralidad de los conocimientos se podrá contribuir eficazmente en la consecución de una educación de calidad e inclusiva, en donde las diversas miradas epistemológicas pueden converger para construir una mejor sociedad. Henao-Villa et al. (2017), apoyan al señalar la importancia que brinda a los estudiantes el aprendizaje basado en proyectos, aunque este se encuentra distanciado del currículo del semestre, lo cual expone como evidencia que aunque se hagan esfuerzos por parte de los docentes por transcender lo mecanicista sin el debido respaldo curricular, será un esfuerzo atomizado que no conducirá a cambios masivos en la población estudiantil, siendo uno de los retos al 2030, la transformación curricular hacia un enfoque centrado en el estudiante, soportado desde la formación pedagógica en las universidades. López-Salazar (2019), colabora indicando la necesidad de estructurar políticas públicas que sean construidas desde las comunidades, diagnosticando sus necesidades, situación similar al de la educación donde surge el escenario de construir desde una episteme donde se involucren los actores educativos en consonancia de edificar currículos contextualizados a sus necesidades e intereses sin perder de vista lo global como escenario de coexistencia colectiva.

El transitar al 2030 es una invitación abierta para configurar nuevos modos de hacer educación. Maldonado (2019), advierte de la metamorfosis de las ciencias sociales, producto de tres razones: "Primera razón: el mundo cambió (p. 115). Segunda razón: aparecen nuevas formas de ver, de explicar, por tanto, nuevos métodos y nuevas técnicas (p. 117). Tercera razón: cambió el ecosistema de las ciencias y el conocimiento" (p. 118). Ignorar lo planteado puede conducir a dejar de lado múltiples posibilidades de hacer ciencia en favor de promover adecuadas acciones en la próxima década, los avances tecnológicos siguen cada día y no existe certeza del mundo en el 2030. Lo cierto es que será distinto al de hoy, 
la diferencia será marcada en cómo se asuma el protagonismo educativo y científico, ojalá sea una cosmovisión epistemológica humanista e integradora de los conocimientos, dejando de lado la dicotomía que propone la supremacía del saber-poder como núcleo estático de conformismo praxiológico de los actores educativos.

\section{Conclusiones}

La revisión bibliométrica realizada vislumbra un horizonte de cara al 2030, basado en una educación diferente a la tradicional, donde se acentúa la necesidad de generar sinergias de epistemologías, métodos, técnicas, en pro de articular una gestión educativa integral donde las diferencias se constituyan en fortalezas por medio de la interrelación intercultural de las personas, saberes, conocimientos, en un marco de respeto mutuo, aceptación de las diversas posturas ideológicas para confluir en un currículo integrador en el cual se trabaje por áreas contextualizadas a la pertinencia social de la glocalidad.

Construir un currículo integrador requiere el aporte protagónico de todos los actores sociales, con la finalidad de edificar en función de lo que se debe aprender (1), de lo que se quiere aprender (2) y lo necesario de aprender (3). Tres distinciones que se diferencian: 1 . Lo que se debe aprender funciona como lo elemental que todos deben aprender. 2. Lo que se quiere aprender es brindarle la oportunidad a los estudiantes de ser escuchados para que participen en la construcción del conocimiento, promoviéndose una sociedad democrática basada en la equidad, respeto, autodeterminación de la persona, como factores primordiales para configurar un ciudadano ético, participativo, crítico y reflexivo, en los diversos procesos socioeconómicos a los cuales se enfrenta día a día. 3. Lo que se quiere aprender es lo que demanda la sociedad, el mundo globalizado para su progreso sostenible, así un estudiante que se encuentre en el medio rural podrá emprender acciones en función de aprender a trabajar con los recursos disponibles en su contexto.

Así el currículo educativo procurará trabajar en la conjunción de conocimientos científicos, saberes ancestrales y prácticas tecnológicas como un conjunto necesario para promover el buen vivir de la ciudadanía, evitándose la exclusión en toda su extensión humana y social. De ese modo, el currículo proyecta un ciudadano con visión global sostenible, por lo que es necesario que las instituciones educativas se redescubran para tal fin, por cuanto el modo de gerenciar en la educación debe girar con base en la fundación de una epistemología muti, inter, transdisciplinaria, compleja, sistémica y holística, necesitándose para tal fin formar a los docentes activos en todos los niveles del sistema educativo, futuros profesionales de la docencia, por lo cual las universidades están llamadas a aportar hoy en el cambio del mañana.

Lo planteado no se puede lograr si no hay una recta participación de los actores políticos, empresariales, sociales, culturales y deportivos, de la nación, por cuanto se necesita del aporte de todos para construir políticas educativas donde cada uno perciba su aporte, generándose identificación con un currículo educativo de cara al 2030, aunado a la inversión económica necesaria para la educación y su sostenimiento como servicio de calidad a la sociedad. Es necesario promover la gratuidad de la educación, pero no la regalía de la misma —es decir- los actores sociales deben comprender el gran valor de la educación, alejándose de posturas populistas que perciben la educación como un conjunto de votos y no de ciudadanía pensante, crítica y reflexiva.

Latinoamérica si realmente quiere crecer, progresar, y dejar de ser el continente de la esperanza para ser el protagonista global, debe confluir en una educación verdaderamente crítica, productiva y sostenible, esto implica amplificar en el currículo la perspectiva del emprendimiento, derogándose la postura del asalariado. No representa significancia similar asistir a la escuela con el convencimiento de formarte para ser asalariado 
algún día que el de producir e innovar para transformar la sociedad. Es allí donde la visión hacia la investigación podría ir girando a estilos donde se promueva la invención sostenible para la productividad de la glocalidad, es promover una economía diversificada no dependiente exclusivamente del patrón Estado, siendo necesario promover la ética como factor transversal para contribuir en la constitución de personas éticas, humanistas y cooperativas, en la construcción de alianzas para el sostenimiento ecológico del ecosistema social donde se desenvuelven.

\section{Referencias bibliográficas}

Acosta, J. (2016). Interdisciplinariedad y transdisciplinariedad: perspectivas para la concepción de la universidad por venir. [Interdisciplinarity and transdisciplinarity: perspectives for the conception of the university to come]. Alteridad, 11(2), 148-156. https://doi.org/10.17163/alt.v11n2.2016.01

Agosto, G., Fontela, M., Brandy, L., \& Langsam, M. (2018). La Agenda 2030 como herramienta de desarrollo para los jóvenes en Argentina. [The 2030 Agenda as a development tool for young people in Argentina]. Ciência \& Saúde Coletiva, 23(9), 2797-2802.

https://dx.doi.org/10.1590/1413-81232018239.13472018

Aldana-Zavala, J.J. (2019). La competencia epistemológica en el investigador social universitario venezolano. [Epistemological competence in the Venezuelan university social researcher]. Praxis, 15(1), 103-115. https://bit.ly/390Omwc

Aldana-Zavala, J., \& Colina-Ysea, F. (2019). Marketing verde en la conformación de una ciudadanía planetaria en el ámbito educativo latinoamericano. [Green marketing in the formation of a planetary citizenship in the Latin American educational field] Revista San Gregorio, 0(31), 150-161. https://bit.ly/392bmLo

Alfaro-Mardones, J., Fernández-Hernández, C., \& González-García, M. (2015). La transdisciplinariedad una herramienta para apuntar al Buen Vivir. [Transdisciplinarity a tool to target Good Living]. Polis (Santiago), 14(40), 23-42. https://dx.doi.org/10.4067/S0718-65682015000100002
Aya-Velandia, L. (2018). Cerebro-complejidad y bioresistencia. [Brain-Complexity and Bioresistance]. Revista de Investigación Psicológica, (19), 195-206. https://n9.cl/dd073

Azorín-Abellán, C. (2017). Una mirada desde los organismos internacionales a la educación para todos. [A look from international organizations to education for all]. Opción, 33(83), 203-228. https://bit.ly/3b6T90u

Borrero, R. (2019). Formación investigativa de los docentes en las instituciones públicas de Educación Secundaria: Un abordaje desde la gerencia. [Investigative Training of teachers in Public Secondary Education Institutions: An Approach from Management]. Revista Arbitrada Interdisciplinaria Koinonía, 4(8), 544-570. http://dx.doi.org/10.35381/r.k.v4i8.298

Buendía-Arias, X.P., Zambrano-Castillo, L.C., \& Insuasty, E.A. (2018). El desarrollo de competencias investigativas de los docentes en formación en el contexto de la práctica pedagógica. [The development of investigative skills of teachers in training in the context of pedagogical practice]. Folios, (47), 179-195. https://doi.org/10.17227/folios.47-7405

Calvo, G. (2013). La formación de docentes para la inclusión educativa. [Teacher training for educational inclusion]. Páginas de Educación, 6(1), 19-35. https://bit.ly/2x6fxIG

Carrillo-Flores, I. (2016). Balance de los Objetivos de Desarrollo del Milenio y su impacto en el derecho a la educación. [Taking stock of the Millennium Development Goals and their impact on the right to education]. Educación, 25(49), 103-119. https://dx.doi.org/10.18800/educacion.201602.006

Collado-Ruano, J. (2016). La huella socioecológica de la globalización. [The socio-ecological footprint of globalization]. Sociedad y Ambiente, (11), 92-121. https://n9.cl/gtyz3

Collado-Ruano, J. (2017a). Interculturalidad y descolonialidad: Retos y desafíos epistemológicos. [Interculturality and decoloniality: Epistemological challenges and challenges]. Revista nuestrAmérica, 5(9), 38-57. https://bit.ly/2Ui3lMT 
Collado-Ruano, J. (2017b). Educación y desarrollo sostenible: la creatividad de la naturaleza para innovar en la formación humana. [Education and sustainable development: the creativity of nature to innovate in human formation]. Educación y Educadores, 20(2), 229-248. https://bit.ly/2xNbPUW

Collado-Ruano, J., Madroñero-Morillo, M., \& ÁlvarezGonzález, F. (2018). Educación transdisciplinar: formando en competencias para el buen vivir. [Transdisciplinary education: training in skills for good living]. Ensaio: Avaliação e Políticas Públicas em Educação, 26(100), 619-644. https://dx.doi.org/10.1590/s0104-40362018002601487

Cornejo-Espejo, J. (2019). Nuevos excluidos en el sistema educacional chileno: problemas y desafíos. [New excluded in the Chilean educational system: problems and challenges] Páginas de Educación, 12(1), 28-48. https://dx.doi.org/10.22235/pe.v12i1.1766

Cosme-Casulo, J. (2018). Los Objetivos de Desarrollo Sostenible y la academia. [The Sustainable Development Goals and the academy]. MEDISAN, 22(8), 838-848. https://n9.cl/0ovgo

Cúnico, S., Pizzinato, A., Strey, M., \& Manso, A. (2018). Desafíos y posibilidades de la investigación cualitativa en Psicología: problematizaciones necesarias. Challenges and possibilities of qualitative research in Psychology: necessary problematizations]. Psicología, Conocimiento y Sociedad, 8(1), 194-217. https://dx.doi.org/10.26864/pcs.v8.n1.9

Del Arco-Bravo, I., Flores-Alarcia, O, \& Silva, P. (2019). El desarrollo del modelo flipped classroom en la universidad: impacto de su implementación desde la voz del estudiantado. [The development of the flipped classroom model in the university: impact of its implementation from the voice of the student body]. Revista de Investigación Educativa, 37(2), 451-469. http://dx.doi. org/10.6018/rie.37.2.327831

Díaz-Bazo, C. del P. (2017). La investigación-acción en la educación básica en Iberoamérica. Una revisión de la literatura. [Action research in basic education in Latin America. A review of the literature]. Magis, Revista Internacional de
Investigación en Educación, 10(20), 159-182. https://doi.org/10.11144/Javeriana.m10-20.iaeb

Escámez-Sánchez, J., Peris-Cancio, J., \& EscámezMarsilla, J. (2017). Educación de los estudiantes universitarios y gestión de la sostenibilidad. [University student education and sustainability management]. Perfiles educativos, 39(156), 174-190. https://n9.cl/o6y4

Fernández-Galindez, O. (2019). La transcomplejidad, una visión epistemológica del siglo XXI. [Transcomplexity, an epistemological vision of the 21st century]. Revista Conocimiento Libre y Licenciamiento CLIC, 19(10), 101116. https://bit.ly/2UcFjTL

Fernández-Hernández, S., \& Cárdenas-Berrio, M. (2015). Formación de competencias investigativas en Ciencias de la Información desde la Universidad de La Habana. [Training of investigative skills in Information Sciences from the University of Havana]. Revista Cubana de Información en Ciencias de la Salud, 26(1), 34-47. https://bit.ly/2WlwSIr

García, N., Paca, N., Arista, S., Valdez, B., \& Gómez, I. (2018). Investigación formativa en el desarrollo de habilidades comunicativas e investigativas. [Formative research in the development of communication and research skills]. Revista de Investigaciones Altoandinas, 20(1), 125-136. https://dx.doi.org/10.18271/ria.2018.336

García-Garnica, M., \& Martínez-Garrido, C. (2019). Dirección escolar y liderazgo en el ámbito iberoamericano. [School Management and Leadership in the Ibero-American Area]. Profesorado, Revista de Currículum y Formación del Profesorado, 23(2), 1-11. https://dx.doi.org/10.30827/profesorado.v23i2.9690

García-Gutiérrez, Z., \& Aznar-Díaz, I. (2019). The Development of Research Competencies, an Alternative to Train Childhood Educators as Teacher-Researchers. [El desarrollo de competencias de investigación, una alternativa para capacitar a educadores de la infancia como docentes-investigadores] Revista Electrónica Educare, 23(1), 1-22. https://doi.org/10.15359/ree.23-1.15

Henao-Villa, C., García-Arango, D., Aguirre-Mesa, E., González-García, A., Bracho-Aconcha, R., Solórzano-Movilla, J., \& Arboleda-López, 
A. (2017). Multidisciplinariedad, interdisciplinariedad y transdisciplinariedad en la formación para la investigación en ingeniería. [Multidisciplinarity, interdisciplinarity and transdisciplinarity in training for engineering research]. Revista Lasallista de Investigación, 14(1), 179-197. https://dx.doi.org/10.22507/rli.v14n1a16

Huapaya-Capcha, Y. (2019). Gestión por procesos hacia la calidad educativa en el Perú. [Process management towards educational quality in Peru]. Revista Arbitrada Interdisciplinaria Koinonía, 4(8), 243-261. http://dx.doi.org/10.35381/r.k.v4i8.277

Huchim-Aguilar, D., \& Reyes-Chávez, R. (2013). La investigación biográfico-narrativa, una alternativa para el estudio de los docentes. [Biographical-narrative research, an alternative for the study of teachers]. Revista Actualidades Investigativas en Educación, 13(3), 1-27. https://doi.org/10.15517/aie. v13i3.12026

Izquierdo-Rus, T., Asensio-Martínez, E., EscarbajalFrutos, A., \& Rodríguez-Moreno, J. (2019). El aprendizaje cooperativo en la formación de maestros de Educación Primaria. [Cooperative learning in the training of primary education teachers]. Revista de Investigación Educativa, 37(2), 543-559. http://dx.doi.org/10.6018/rie.37.2.369731

Locatelli, R. (2018). La educación como bien público y común. Reformular la gobernanza de la educación en un contexto cambiante. [Education as a public and common good. Reformulating the governance of education in a changing context]. Perfiles educativos, 40(162), 178-196. https://bit.ly/3ab5qAY

Loli-Ponce, R., Sandoval-Vegas, M., Ramírez-Miranda, E., Quiroz-Vásquez, M., Navarro-Casquero, R., \& Rivas-Díaz, L. (2015). La enseñanza aprendizaje de la investigación: representación social desde la perspectiva estudiantil. [Teaching learning research: social representation from the student perspective]. Anales de la Facultad de Medicina, 76(1), 47-56. https://n9.cl/x1dzh

López-Salazar, R. (2019). Bienestar y desarrollo: evolución de dos conceptos asociados al bien vivir. [Well-being and development: evolu- tion of two concepts associated with living well]. Revista Telos, URBE, Ciencias Sociales, 21(2), 288-312. https://doi.org/10.36390/ telos 212.03

Maldonado, C. (2019). Tres razones de la metamorfosis de las ciencias sociales en el siglo XXI. [Three reasons for the metamorphosis of the social sciences in the 21st century]. Cinta de Moebio. Revista de Epistemología de Ciencias Sociales, (64), 114-122. https://bit.ly/33r3vpu Maya, E., Aldana-Zavala, J., \& Isea-Argüelles, J. (2019). Liderazgo directivo y educación de calidad. [Management Leadership and Quality Education]. CIENCIAMATRIA, 5(9), 114129. https://doi.org/10.35381/cm.v5i9.102

Meléndez-Rojas, R. (2017). Prospectiva para la educación costarricense. Una oportunidad de transformar el sistema educativo para el siglo XXI. [Prospective for Costa Rican education. An opportunity to transform the educational system for the 21st century]. Actualidades Investigativas en Educación, 17(3), 720-744. https://dx.doi.org/10.15517/aie.v17i3.29072

Molina-Naranjo, J., Lavandero-García, J., \& Hernández-Rabell, L. (2018). El modelo educativo como fundamento del accionar universitario.: Experiencia de la Universidad Técnica de Manabí, Ecuador. [The educational model as the foundation of university action: Experience of the Technical University of Manabí, Ecuador]. Revista Cubana de Educación Superior, 37(2), 151-164. https://n9.cl/bvikz

Morgado, C., Aldana-Zavala, J., \& Isea-Argüelles, J. (2019). Gerencia transformacional desde el accionar docente directivo para el abordaje del contexto escolar. [Transformational management from the directive teaching action to approach the School Context]. CIENCIAMATRIA, 5(9), 48-72. https://doi.org/10.35381/cm.v5i9.99

Muñoz-Martínez, M., \& Garay-Garay, F. (2015). La investigación como forma de desarrollo profesional docente: Retos y perspectivas. [Research as a form of teacher professional development: Challenges and perspectives]. Estudios pedagógicos (Valdivia), 41(2), 389-399. https://dx.doi.org/10.4067/S0718-07052015000200023 
Murillo, F., \& Duk, C. (2017). El ODS 4 (y el 16) como meta para los próximos años. [SDG 4 (and 16) as a goal for the coming years]. Revista Latinoamericana de Educación Inclusiva, 11(2), 11-13. https://dx.doi.org/10.4067/S0718-73782017000200001

Murillo, F., \& Martínez-Garrido, C. (2019). Una mirada a la investigación educativa en América Latina a partir de sus artículos. [A Look at Educational Research in Latin America from its Articles]. REICE. Revista Iberoamericana sobre Calidad, Eficacia $y$ Cambio en Educación, 17(2). http://dx.doi.org/10.15366/reice2019.17.2.001

Peche-Cruz, H., \& Giraldo-Supo, V. (2019). El Aprendizaje Flip Learning centrado en el estudiante como generador de calidad educativa. [Student-centered Flip Learning as a generator of educational quality]. Revista Arbitrada Interdisciplinaria Koinonía, 4(8), 427-450. http://dx.doi.org/10.35381/r.k.v4i8.293

Pérez, L. (2017). Desarrollo de capacidades investigativas en estudiantes de pregrado de la carrera de arquitectura de la Facultad de Arquitectura y Urbanismo de la Universidad Técnica de Oruro: Resultados del estudio de campo. [Development of investigative capacities in undergraduate students of the architecture degree of the Faculty of Architecture and Urbanism of the Technical University of Oruro: Results of the field study]. Revista Ciencia, Tecnología e Innovación, 14(15), 861-868. https://n9.cl/3zb6

Pérez-Van Leenden, M. (2019). La investigación acción en la práctica docente. Un análisis bibliométrico (2003-2017). [Action research in teaching practice. A bibliometric analysis (2003-2017)]. magis, Revista Internacional de
Investigación en Educación, 12(24), 177-192. https://doi.org/10.11144/Javeriana.m10-20.ncev

Proestakis-Maturana, A., \& Terrazas-Núñez, W. (2017). Formación en investigación y supervisión en programas de doctorados. [Research training and supervision in doctoral programs]. Magis, Revista Internacional de Investigación en Educación, 10(20), 85-104. https://doi.org/10.11144/Javeriana.m10-20.fisp

Rivas-Tovar, L. (2011). Las nueve competencias de un investigador. [The nine skills of an investigator]. Investigación Administrativa, 108(2), 34-54. https://bit.ly/3diaXYK

Rodríguez-Fiallos, J., Navarrete-Pita, Y., EstradaMolina, O., \& Vera-Viteri, L. (2019). Valoraciones acerca de la relación entre competencias profesionales y las investigativas. [Assessments about the relationship between professional and investigative skills]. Revista Cubana de Educación Superior, 38(1), 5-11. https://n9.cl/6l2jg

UNESCO (2005). Hacia las sociedades del conocimiento. https://bit.ly/39Ydll7

Vera-Del Carpio, R. (2015). Gestión prospectiva sobre las dimensiones de desarrollo sostenible hacia el 2030 en los líderes de la Región de Puno-Perú. [Prospective management on the dimensions of sustainable development towards 2030 in the leaders of the Puno Region-Peru] Comuni@cción, 6(1), 05-15. https://n9.cl/w718

Villa-Sánchez, A. (2019). Liderazgo: una clave para la innovación y el cambio educativo. [Leadership: a key to innovation and educational change]. Revista de Investigación Educativa, 37(2), 301-326. http://dx.doi.org/10.6018/rie.37.2.365461 